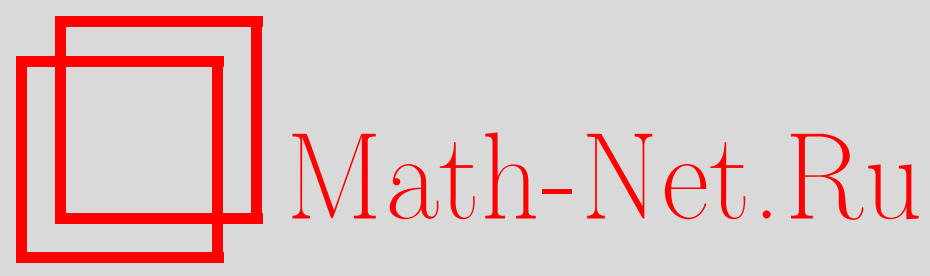

В. А. Скворцов, А. П. Солодов, Об одном обобщении интеграла Данжуа, Матем. заметки, 1997, том 62, выпуск 5, 766-772

DOI: https://doi.org/10.4213/mzm1662

Использование Общероссийского математического портала Math-Net.Ru подразумевает, что вы прочитали и согласны с пользовательским соглашением http://www.mathnet.ru/rus/agreement

Параметры загрузки:

IP: 107.22 .136 .117

26 апреля 2023 г., 14:52:17

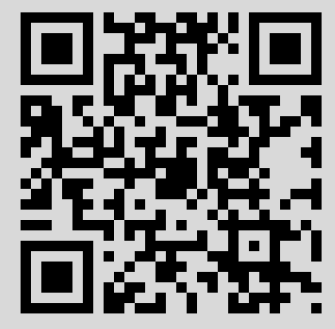




\title{
ОБ ОДНОМ ОБОБЩЕНИИ ИНТЕГРАЛА ДАНЖУА
}

\author{
В. А. Скворцов, А. П. Солодов
}

\begin{abstract}
Доказьвается, что $M$-интеграл Изеки является существенно более общим, чем широкий интеграл Данжуа.

Библиограффия: 6 названий.
\end{abstract}

В данной заметке рассматривается введенный Изеки $M$-интеграл (см. [1]) и дается положительньй ответ на вопрос Изеки, является ли $M$-интеграл существенно более обшим, чем широкий интеграл Данжуа.

Определение Изеки базируется на понятии стабильности функции в некотором классе $\mathscr{P}$, являющемся подмножеством класса $\mathrm{ADN}$ - класса всех аппроксимативно дифференцируемых непрерывных функиий, обладающих $N$-свойством Лузина, т.е. отображаюших всякое множество меры 0 в множество меры 0 .

ОПРедЕЛЕниЕ 1. Функция $F:[a, b] \rightarrow \mathbb{R}$ называется стабильной в некотором классе $\mathscr{P} \subset \mathrm{ADN}$, если она принадлежит $\mathscr{P}$ и для любой другой функции $G \in \mathscr{P}$ из условия $F_{a p}^{\prime}(x)=G_{a p}^{\prime}(x)$ п.в. на $[a, b]$ следует, что $F-G=$ const.

Другими словами, стабильная функция является единственной внутри класса $\mathscr{P}$ (с точностью до аддитивной постоянной) первообразной для своей аппроксимативной производной. Поэтому понятие стабильности может быть использовано для дескриптивного определения интеграла.

Изеки в [1] рассматривал два таких определения. В первом случае в качестве $\mathscr{P}$ берется весь класс $\mathrm{ADN}$, что приводит к определению $N$-интеграла.

ОПРЕДЕЛЕнИЕ 2 . Функция $f:[a, b] \rightarrow \mathbb{R}$ называется $N$-интегрируемой, если существует стабильная в $\mathrm{ADN}$ функция $F$ такая, что $F_{a p}^{\prime}(x)=f(x)$ п.в. на $[a, b]$.

Второе определение интеграла вводится в [1] на основе понятия $M$-непрерьвности.

ОпредЕЛЕниЕ 3 . Непрерывная функция $F:[a, b] \rightarrow \mathbb{R}$ называется $M$-непрерывной на множестве $E \subset[a, b]$, если для каждого множества $S \subset E$ меры 0 и для любого $\varepsilon>0$ найдется такая последовательность неперекрываюшихся отрезков $\left\{I_{n}\right\}_{n \in \mathbb{N}}$, что $\bigcup_{n} I_{n} \supset S$ и $\sum_{n} \omega\left(F, I_{n} \cap S\right)<\varepsilon$ (здесь $\omega(F, X)$ обозначает колебание функции $F$ на множестве $X)$.

Работа выполнена при поддержке Российского фонда фундаментальных исследований, грант № 96-01-00332. Работа первого автора вьполнена при частичной поддержке фонда INTAS, грант № 94-1044. 
ОпредЕлЕниЕ 4. Непрерьвная функция $F:[a, b] \rightarrow \mathbb{R}$ назьвается обобщенно $M$-не-

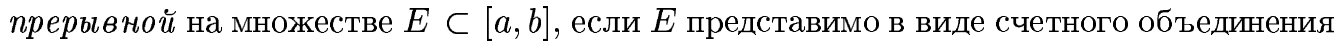
множеств, на каждом из которьх функция $F$ является $M$-непрерывной.

(В [2] классы $M$-непрерывных и обобщенно $M$-непрерывных функций обозначаются соответственно через $\mathscr{E}_{1}$ и $\mathscr{E}_{1} G$.)

Класс всех обобщенно $M$-непрерывных аппроксимативно дифференцируемых функций обозначим через ADM. Ясно, что ADM $\subset$ ADN.

ЗАмЕчАниЕ. Если функция $F$ является $M$-непрерывной на каждом из множеств $E_{1}$ и $E_{2}$, то она $M$-непрерьвна и на их объединении. Далее, пусть функция $F$ обобщенно $M$-непрерьвна на $E$, т.е.

$$
E=\bigcup_{n=1}^{\infty} E_{n}
$$

и $F$ является $M$-непрерьвной на каждом $E_{n}$. Положим

$$
E_{n}^{\prime}=\bigcup_{k=1}^{n} E_{k} .
$$

Тогда $F$ будет $M$-непрерьвной на каждом $E_{n}^{\prime}$, и для каждого $n$ справедливо $E_{n}^{\prime} \subset E_{n+1}^{\prime}$.

ОПРЕДЕЛЕНИЕ 5. Функция $f:[a, b] \rightarrow \mathbb{R}$ называется $M$-интегрируемой, если существует стабильная в $\mathrm{ADM}$ функция $F$ такая, что $F_{a p}^{\prime}(x)=f(x)$ п.в. на $[a, b]$.

Напомним также определение широкого интеграла Данжуа (см. [3]).

ОПРЕДЕЛЕНИЕ 6. Функция $F:[a, b] \rightarrow \mathbb{R}$ назьвается АС-функиией на множестве $E \subset[a, b]$, если для любого $\varepsilon>0$ существует $\delta>0$ такое, что для любой последовательности неперекрывающихся отрезков $\left\{\left[a_{n}, b_{n}\right]\right\}_{n \in \mathbb{N}}$, концы которых принадлежат $E$, из условия $\sum_{n}\left|b_{n}-a_{n}\right|<\delta$ следует, что $\sum_{n}\left|F\left(b_{n}\right)-F\left(a_{n}\right)\right|<\varepsilon$.

ОпредЕЛЕниЕ 7. Непрерывная функция $F:[a, b] \rightarrow \mathbb{R}$ называется ACG-функиией на множестве $E \subset[a, b]$, если $E$ представимо в виде счетного объединения множеств, на каждом из которых $F$ является АС-функцией.

ОПРЕДЕЛЕНИЕ 8. Функция $f:[a, b] \rightarrow \mathbb{R}$ называется $D$-интегрируемой, если существует ACG-функция $F$ такая, что $F_{a p}^{\prime}(x)=f(x)$ п.в. на $[a, b]$.

Легко проверить (см. [1] и [4]), что ACG $\subsetneq \mathrm{ADM} \subsetneq \mathrm{ADN}$. Известно (см. [5]), что всякая ACG-функция является стабильной в классе ADN и тем более в классе ADM. Поэтому $D$-интеграл не уже $N$-интеграла и $M$-интеграла. Изеки в [1] поставил вопрос о том, является ли включение $D$-интеграла в эти интегралы строгим. Что касается $N$-интеграла, то на этот вопрос отвечает следующая теорема, доказанная в [6].

ТеоремА А. Пусть функиия $F$ непрерывна на отрезке $[a, b]$, обладает $N$-свойством и почти всюду аппроксимативно дифферениируема. Тогда $F$ является ACG-функиией тогда и только тогда, когда любая другая непрерывная, обладающая $N$-свойством, почти всюду аппроксимативно дифференцируемая функция $G$, у которой $G_{a p}^{\prime}(x)=F_{a p}^{\prime}(x)$ n.в., отличается от $F$ на аддитивную постоянную.

Иначе говоря, $N$-интеграл в точности совпадает с $D$-интегралом.

Здесь мы покажем, что в отличие от $N$-интеграла $M$-интеграл оказьвается существенно более общим, чем $D$-интеграл. Это будет вытекать из следующей теоремы. 
TеоремА. Существует функция $F$, стабильная в $\mathrm{ADM}$, но не являющаяся ACG-функиией.

ДокАЗАТЕЛЬСтво. Пусть $C$ - канторово совершенное множество на $[0,1]$ и пусть $\Delta_{k}^{r}=\left(a_{k}^{r}, b_{k}^{r}\right), 1 \leqslant k \leqslant 2^{r-1}, r=1,2, \ldots,-$ смежные интервалы длины $1 / 3^{r}$. Обозначим через $I_{j}^{n}, 1 \leqslant j \leqslant 2^{2^{n}}$, интервалы, составляющие множество

$$
(0,1) \backslash \bigcup_{m=0}^{n} \bigcup_{k=1}^{2^{2^{m}}-1}\left[a_{k}^{2^{m}}, b_{k}^{2^{m}}\right]
$$

Каждая точка $x \in[0,1)$ единственньм образом представляется в виде

$$
x=\sum_{i=1}^{\infty} \frac{x_{i}}{3^{i}}, \quad x_{i}=0,1 \text { или } 2
$$

(условимся для троично-рациональных точек использовать представление в виде конечной дроби).

Заметим, что коэффициент $x_{2^{n}}$ постоянен на каждом интервале $I_{j}^{n}$, принимая значения 0 или 2, и положим для каждого $n=2,3,4, \ldots$

$$
f_{n}(x)=\frac{x_{2^{n}}}{2^{2^{n}}}, \quad x \in I_{j}^{n}, \quad 1 \leqslant j \leqslant 2^{2^{n}} .
$$

Доопределим $f_{n}$ на замыканиях интервалов $I_{j}^{n}$ по непрерьвности, а затем на интервалах $\Delta_{k}^{2^{m}}, 1 \leqslant k \leqslant 2^{2^{m}-1}, 0 \leqslant m \leqslant n$, линейно так, чтобы $f_{n}$ была непрерьвна на [0, 1$]$. Заметим, что при $k>n$

$$
\omega\left(f_{k}, I_{j}^{n}\right)=2^{1-2^{k}} .
$$

Кроме того, $\left|f_{n}(x)\right| \leqslant 2^{1-2^{n}}$ при всех $x \in[0,1]$. Поэтому ряд

$$
\sum_{n=2}^{\infty} f_{n}(x)
$$

сходится равномерно и функция

$$
F(x)=\sum_{n=2}^{\infty} f_{n}(x)
$$

непрерьвна на $[0,1]$. Заметим для дальнейшего, что

$$
\left|F\left(b_{k}^{2^{n}}\right)-F\left(a_{k}^{2^{n}}\right)\right| \geqslant \frac{1}{2^{2^{n}}} .
$$

Отметим также следующее свойство функции $F$.

Лемма. Для любы $x, y \in C$ неравенство $|F(x)-F(y)| \leqslant 3 / 2^{2^{n}+1}$ обеспечивает более сильное неравенство $|F(x)-F(y)|<3 / 2^{2^{n+1}}$. 
ДокАЗАТЕльство. Зафиксируем $x$ и $y$, для которых выполнены условия леммы, и покажем, что $f_{m}(x)=f_{m}(y)$ при всех $m, 2 \leqslant m \leqslant n$. Если бы это было не так, то, взяв наименьшее из тех $m$, для которых $f_{m}(x) \neq f_{m}(y)$, мы получили бы $\left|f_{m}(x)-f_{m}(y)\right|=$ $2 / 2^{2^{m}}$ и

$$
\begin{aligned}
|F(x)-F(y)| & \geqslant \frac{2}{2^{2^{m}}}-\sum_{k=m+1}^{\infty}\left|f_{k}(x)-f_{k}(y)\right| \geqslant \frac{2}{2^{2^{m}}}-\sum_{k=m+1}^{\infty} \frac{2}{2^{2^{k}}} \\
& >\frac{2}{2^{2^{m}}}-\frac{4}{2^{2^{m+1}}}>\frac{3}{2^{2^{m}+1}} \geqslant \frac{3}{2^{2^{n}+1}},
\end{aligned}
$$

что противоречит условию. Таким образом, $f_{m}(x)=f_{m}(y)$ для всех $m, 2 \leqslant m \leqslant n$, и стало быть,

$$
|F(x)-F(y)| \leqslant \sum_{k=n+1}^{\infty}\left|f_{k}(x)-f_{k}(y)\right| \leqslant \sum_{k=n+1}^{\infty} \frac{2}{2^{2^{k}}}<\frac{3}{2^{2^{n+1}}},
$$

что и доказьвает лемму.

Проверим теперь, что $F$ обобщенно $M$-непрерывнана $[0,1]$. Так как $F$ линейна на $\Delta_{k}^{r}$, $1 \leqslant k \leqslant 2^{r-1}$, то она $M$-непрерьвна на $\Delta_{k}^{r}$. Следовательно, осталось показать, что $F$ является $M$-непрерывной на $C$.

Ясно, что

$$
\bigcup_{j=1}^{2^{2^{n}}} \bar{I}_{j}^{n} \supset C
$$

$(\bar{X}$ означает замыкание множества $X)$. Задав произвольное $\varepsilon>0$, мы можем выбрать $n$ так, что $2^{2-2^{n}}<\varepsilon$. Тогда с учетом $(1)$

$$
\sum_{j=1}^{2^{2^{n}}} \omega\left(F, \bar{I}_{j}^{n} \cap C\right) \leqslant \sum_{j=1}^{2^{2^{n}}} \omega\left(F, \bar{I}_{j}^{n}\right) \leqslant 2^{2^{n}} \sum_{k=n+1}^{\infty} \frac{2}{2^{2^{k}}} \leqslant \frac{2^{2^{n}}}{2^{2^{n+1}-2}}=2^{2-2^{n}}<\varepsilon
$$

Итак, $F$ обобщенно $M$-непрерывна.

Покажем, что для любой функции $G$, принадлежащей классу ADM, из условия $G_{a p}^{\prime}(x)=F_{a p}^{\prime}(x)$ п.в. на $[0,1]$ следует, что $G-F=$ const. Пусть это не так. Пусть $S$ - множество точек, ни в какой окрестности каждой из которых непрерывная функция $H=G-F$ не является постоянной. По предположению $S$ непусто. Ясно, что $S-$ совершенное множество, на смежных интервалах которого $H$ постоянна. На смежных интервалах множества $C$ функция $F$ линейна и, стало быть, ACG. Теорема A показывает, что $H$ постоянна на этих смежных интервалах, т.е. $S \subset C$. Функция $G$ обобщенно $M$-непрерьвна на $[0,1]$, а значит, и на $S$, т.е. $S$ представимо в виде счетного объединения множеств $S_{n}$, на каждом из которых функция $G$ будет $M$-непрерывной. Согласно замечанию к определению 4 можно считать, что $S_{n} \subset S_{n+1}$. По теореме Бэра о категориях найдется порция $[\alpha, \beta] \cap S,(\alpha, \beta) \cap S \neq \varnothing$, а также номер $N$ такие, что для любого $n \geqslant N$ множество $[\alpha, \beta] \cap S_{n}$ всюду плотно в $[\alpha, \beta] \cap S$. Так как $(\alpha, \beta) \cap S \neq \varnothing$, функция $H$ не является постоянной на отрезке $[\alpha, \beta]$, поэтому $|H([\alpha, \beta] \cap S)| \neq 0$ (здесь 
$f(X)$ обозначает образ множества $X$ при отображении $f$, а $|X|$ - меру множества $X)$. Отсюда и из равенства

$$
H([\alpha, \beta] \cap S)=\bigcup_{n=N}^{\infty} H\left([\alpha, \beta] \cap S_{n}\right)
$$

вытекает, что для некоторого $n \geqslant N$ выполнено $\left|H\left([\alpha, \beta] \cap S_{n}\right)\right| \neq 0$. Обозначим через $S^{\prime}$ множество $[\alpha, \beta] \cap S_{n}$, где $n$ выбрано выше. Итак, функция $G$ является $M$-непрерывной на множестве $S^{\prime}$, которое всюду плотно в $[\alpha, \beta] \cap S$, и при этом $\left|H\left(S^{\prime}\right)\right| \neq 0$.

Рассмотрим произвольньй отрезок $I$ такой, что $I \cap S^{\prime} \neq \varnothing$. Положим

$$
A=\min _{x \in I \cap \overline{S^{\prime}}} F(x), \quad B=\max _{x \in I \cap \overline{S^{\prime}}} F(x)
$$

Минимум и максимум достигаются в точках $\bar{x}$ и $\bar{y}$ соответственно. Предположим сначала, что $A<B$. Не ограничивая общности, будем считать, что $\bar{x}<\bar{y}$. Заметим, что $B-A \leqslant \omega(F,[0,1]) \leqslant 1 / 4$, и найдем натуральное $m \geqslant 2$, для которого вьполнены неравенства

$$
\begin{gathered}
B-A \geqslant \frac{3}{2^{2^{m}+1}}, \\
B-A<\frac{3}{2^{2^{m-1}+1}} .
\end{gathered}
$$

Лемма позволяет усилить неравенство (4):

$$
B-A<\frac{3}{2^{2^{m}}}
$$

Далее, пусть

$$
\begin{aligned}
& x^{*}=\sup \left\{x: x<\bar{y}, x \in I \cap S^{\prime}, B-F(x) \geqslant \frac{3}{2^{2^{m}+1}}\right\}, \\
& y^{*}=\inf \left\{y: y>x^{*}, y \in I \cap S^{\prime}, F(y)-A \geqslant \frac{3}{2^{2^{m}+1}}\right\} .
\end{aligned}
$$

Заметим, что $x^{*}<y^{*}$, так как в противном случае выполняется неравенство $B-A \geqslant$ $3 / 2^{2^{m}}$, противоречащее (5). Интервал $\left(x^{*}, y^{*}\right)$ не содержит точек множества $S^{\prime}$. Действительно, если $z \in\left(x^{*}, y^{*}\right) \cap S^{\prime}$, то из определения $x^{*}$ следует, что $B-F(z)<3 / 2^{2^{m}+1}$. Тогда согласно лемме

$$
B-F(z)<\frac{3}{2^{2^{m+1}}} .
$$

Аналогично, из определения $y^{*}$ и леммы следует, что

$$
F(z)-A<\frac{3}{2^{2^{m+1}}} .
$$

Из (6) и (7) вытекает, что $B-A<6 / 2^{2^{m+1}} \leqslant 3 / 2^{2^{m}+1}$. Получаем противоречие с (3). 
Таким образом, $S^{\prime} \cap\left(x^{*}, y^{*}\right)=\varnothing$, откуда $S \cap\left(x^{*}, y^{*}\right)=\varnothing$. Поэтому функция $H$ постоянна на $\left[x^{*}, y^{*}\right]$, и значит,

$$
\omega\left(G,\left[x^{*}, y^{*}\right]\right)=\omega\left(F,\left[x^{*}, y^{*}\right]\right) .
$$

В силу непрерывности функции $G$ и определения точек $x^{*}$ и $y^{*}$ найдутся точки $u, v \in$ $I \cap S^{\prime}$ такие, что

$$
|G(u)-G(v)| \geqslant \frac{1}{2} \omega\left(G,\left[x^{*}, y^{*}\right]\right) .
$$

Соотношения $B-F\left(y^{*}\right)<3 / 2^{2^{m}+1}$ и $F\left(x^{*}\right)-A<3 / 2^{2^{m}+1}$, вытекающие из определения $x^{*}$ и $y^{*}$ и неравенства $x^{*}<y^{*}$, вместе с леммой дают нам неравенства

$$
B-F\left(y^{*}\right)<\frac{3}{2^{2^{m+1}}}, \quad F\left(x^{*}\right)-A<\frac{3}{2^{2^{m+1}}} .
$$

Из (10) и (5) следует, что $F\left(y^{*}\right)-F\left(x^{*}\right)>B-A-2 \cdot 3 / 2^{2^{m+1}}>(B-A) / 2$ при $m \geqslant 2$, т.е.

$$
\omega\left(F,\left[x^{*}, y^{*}\right]\right)>\frac{1}{2} \omega\left(F, I \cap S^{\prime}\right) .
$$

Очевидно также, что

$$
\omega\left(G, I \cap S^{\prime}\right) \geqslant|G(u)-G(v)| .
$$

Соотношения (8), (9), (11) и (12) позволяют заключить, что

$$
\omega\left(G, I \cap S^{\prime}\right) \geqslant \frac{1}{4} \omega\left(F, I \cap S^{\prime}\right) .
$$

В случае $A=B$ неравенство (13) очевидно.

Возьмем $\varepsilon=\left|H\left(S^{\prime}\right)\right| / 5$. Функция $G$ является $M$-непрерьвной на $S^{\prime}$, поэтому сушествует такая последовательность неперекрываюшихся отрезков $\left\{I_{n}\right\}_{n \in \mathbb{N}}$, что $\bigcup_{n} I_{n} \supset S^{\prime}$ и

$$
\sum_{n} \omega\left(G, I_{n} \cap S^{\prime}\right)<\varepsilon
$$

Отсюда и из неравенства (13) вытекает, что

$$
\sum_{n} \omega\left(F, I_{n} \cap S^{\prime}\right)<4 \varepsilon
$$

Используя (14) и (15), получаем

$$
\begin{aligned}
\left|H\left(S^{\prime}\right)\right| & \leqslant \sum_{n} \omega\left(H, I_{n} \cap S^{\prime}\right) \leqslant \sum_{n} \omega\left(G, I_{n} \cap S^{\prime}\right)+\sum_{n} \omega\left(F, I_{n} \cap S^{\prime}\right) \\
& <\varepsilon+4 \varepsilon=5 \varepsilon=\left|H\left(S^{\prime}\right)\right| .
\end{aligned}
$$

Полученное противоречие показывает, что множество $S$ пусто, и значит, $F$ стабильна в классе ADM. 
Наконец, покажем, что $F$ не является ACG-функцией. Если это не так, то найдется непустая порция замкнутого множества $C$, на которой $F$ является АС-функцией. Эта порция содержит множество $I_{j}^{m} \cap C$ для некоторых $j$ и $m$. Для каждого $n$ такого, что $2^{n}>m$, рассмотрим набор отрезков $\Delta_{k}^{2^{n}}=\left[a_{k}^{2^{n}}, b_{k}^{2^{n}}\right]$ таких, что $\Delta_{k}^{2^{n}} \subset I_{j}^{m}$. Их число равно $2^{2^{n}} / 2^{2^{m}+1}$. Тогда с учетом (2)

$$
\begin{gathered}
\sum_{k: \Delta_{k}^{2^{n} \subset I_{j}^{m}}}\left|b_{k}^{2^{n}}-a_{k}^{2^{n}}\right|=\frac{1}{2^{2^{m}+1}}\left(\frac{2}{3}\right)^{2^{n}} \\
\sum_{k: \Delta_{k}^{2^{n}} \subset I_{j}^{m}}\left|F\left(b_{k}^{2^{n}}\right)-F\left(a_{k}^{2^{n}}\right)\right| \geqslant \frac{1}{2^{2^{n}}} \cdot \frac{2^{2^{n}}}{2^{2^{m}+1}}=\frac{1}{2^{2^{m}+1}} .
\end{gathered}
$$

Поскольку в этих соотношениях $n$ произвольно, а $m$ фиксировано, то, устремляя $n$ к бесконечности, получаем противоречие с абсолютной непрерьвностью функции $F$.

Тем самым, теорема доказана.

Авторы пользуются случаем поблагодарить профессора Л. Д. Пьяща (Италия), которая привлекла их внимание к рассмотренной здесь задаче.

\section{СПИСОК ЦИТИРОВАННОЙ ЛИТЕРАТУРЫ}

[1] Iseki K. An attempt to generalize the Denjoy integration // Natur. Sci. Rep. Ochanomizu Univ. 1983. V. 34. № 1. P. 19-33.

[2] Ene V. Real Functions - Current Topics. Lecture Notes in Math. V. 1603. Berlin: Spriger, 1995.

[3] Сакс С. Теория интеграла. М.: ИЛ, 1949.

[4] Iseki K. On quasi-Denjoy integration // Proc. Japan Acad. Ser. A. Math. Sci. 1962. V. 38. P. 252-257.

[5] Foran J. Differentiation and Luzin's condition (N) // Real Anal. Exchange. 1977-78. V. 3. №1. P. 34-37.

[6] Bongiorno B., Preiss D. An unusual descriptive definition of integral // Contemp. Math. 1985. V. 42. P. 13-22. 